Ausbildung

Antje Michel*, Frank Heidmann und Constanze Langer

\title{
Design trifft Informationswissenschaft - Eine Diskussion zum Wandel zweier Disziplinen im Kontext der Digitalisierung
}

https://doi.org/10.1515/iwp-2020-2072

Von Januar 2017 bis März 2019 haben wir, Constanze Langer und Frank Hartmann aus dem Fachbereich Design und Antje Michel aus dem Fachbereich Informationswissenschaften im Rahmen des durch den Stifterverband und die Carl-Zeiss-Stiftung geförderten Curriculumentwicklungsprojekts „Design 0815“ zusammengearbeitet. Ein wesentliches Ziel bestand in der Integration von relevanten Kompetenzen in die Curricula der Design Studiengänge, welche die Studierenden auf die digital geprägten Arbeitswelten von morgen bestmöglich vorbereiten. Dabei wurde der Fokus auf die Stärkung der digitalen Kompetenz und Datenkompetenz (Digital / Data Literacy) sowie auf Problemlösungsstrategien (Problem Solving Strategies) gelegt. Im Projekt wurden außerdem Methoden und Formate zur Gestaltung eines partizipativen Curriculum-Entwicklungsprozesses entwickelt. Zudem wurde am Beispiel der Studiengänge am Fachbereich Informationswissenschaften ermittelt, inwiefern sich die erarbeiteten Lehrkonzepte und Methoden der Curriculum-Entwicklung für die Weiterentwicklung anderer Studiengänge der Hochschule übertragen und nutzen lassen.

In diesem Beitrag möchten wir nicht noch einmal das Projekt „Design 0815“vorstellen. Für eine Information über Projektrahmen und Inhalte sei auf die bereits bestehenden Veröffentlichungen verwiesen. ${ }^{1}$ Für die Leserinnen und Le-

1 Michel, A.; Langer, C.; Heidmann, F. (2019). Design 0815 - Curriculumentwicklung in den Design-Disziplinen. In: DUZ-Wissenschaft und Management 2(10), S. 17-20.; Michel, A., Langer, C., Heidmann, F.:

*Kontaktperson: Prof. Dr. Antje Michel, Fachhochschule Potsdam, Fachbereich Informationswissenschaften, Kiepenheueralle 5, 14469 Potsdam, E-Mail: michel@fh-potsdam.de Prof. Dr. Frank Heidmann, Fachhochschule Potsdam, Fachbereich Design, Kiepenheueralle 5, 14469 Potsdam,

E-Mail: heidmann@fh-potsdam.de

Prof. Constanze Langer, Fachhochschule Potsdam, Fachbereich Design, Kiepenheueralle 5, 14469 Potsdam,

E-Mail: langer@fh-potsdam.de ser der IWP erscheint es uns interessanter, einige grundsätzliche Gedanken zur Kooperation von Informationswissenschaft bzw. Informationspraxis mit den Design-Disziplinen zu diskutieren. Diese stark durch unsere jeweiligen subjektiven professionellen Biografien geprägte Meta-Diskussion über Gegenstand, Methoden, Praktiken und Selbstverständnis unserer Disziplinen, über den Wandel der Design-Berufe sowie der Informationsberufe im Kontext von Globalisierung und Digitalisierung sowie über die Frage, ob und wenn ja inwiefern Design und Informationswissenschaft voneinander profitieren könnten, hat uns während unseres gesamten Projekts begleitet. Wir möchten Sie einladen, sich in diesem Beitrag auf ein etwas anderes Format einzulassen und sich einfach zu uns zu setzen in unser stu-

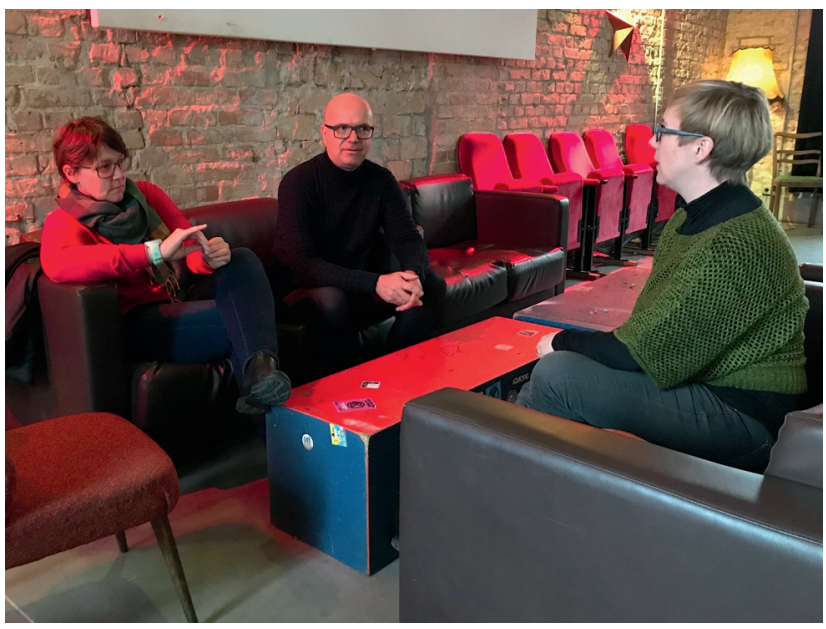

Abbildung 1: Interdisziplinärer Dialog im studentischen Casino der FH Potsdam.

Curriculum design: How can the development of information science curricula benefit from interdisciplinary cooperation? In: Aparac-Jelusic, Tatjana; Casarosa, Vittore; Maceviciute, Elena (Hrsg.), The Future of Education in Information Science. Proceedings from FEIS-International EINFOSE Symposium. 10-11 September 2018 Pisa: Italy. Online verfügbar unter: einfose.ffos.hr/feis-2018/proceedings [18.1.2020]. 
dentisches Casino am Campus der FHP, sich ein unanständig günstiges Getränk zu bestellen und an unserer Diskussion teilzunehmen. Vielleicht beteiligen Sie sich ja sogar aktiv und wir bekommen in einer der nächsten Ausgaben der IWP Ihre Gedanken retour?

\section{Nutzerinnen- und Nutzerzentrierte Informationsaufbereitung}

Constanze Langer: Wenn es so naheliegend ist, dass Design und Informationswissenschaft „irgendwie“ ziemlich viele Berührungspunkte haben bzw. in vielen Kontexten aufeinander angewiesen sind, warum gibt es dann so wenige institutionelle Berührungspunkte - z. B. in den Curricula der beiden Disziplinen, in Form von gemeinsamen Forschungsprojekten etc.? Oder negativ formuliert: warum gibt es so viele Missverständnisse bis hin zu Unverständnis für die jeweils andere Disziplin? Ich nenne ein paar Beispiele für das aufeinander angewiesen sein. Du schreibst, Antje: „Aufgabe der Menschen in Informationsberufen ist es, Informationen aufzubereiten und anderen zu vermitteln."

Wie werden Informationen aufbereitet? Nach wie vor in einem hohen Maße visuell, d.h. neben der textlichen Aufbereitung grafisch, diagrammatisch, illustrativ. Als Bewegtbild, Animation, Simulation, zukünftig vermehrt virtuell oder augmentiert. Die visuelle (und inzwischen auch akustische, haptische, olfaktorische) Aufbereitung von Informationen ist eine Kernkompetenz der Design-Disziplinen, inklusive der Kartografie. Und, jetzt wird es für den interdisziplinären Dialog und die Informationswissenschaft spannend, die Design-Disziplinen verstehen diese Kompetenz nicht mehr allein formal-ästhetisch, sondern auch didaktisch. Wir nennen es User Experience bzw. Usability. Der Fokus ist handlungsorientiert, serviceorientiert, business-orientiert, erlebnis-orientiert; häufig wird Storytelling als Methode verwendet. ${ }^{3}$ Die Dialogprinzipien für die Gestaltung guter Produkte, Informationsmedien und Software sind sehr didaktisch: Aufgabenangemessenheit, Selbstbeschreibungsfähigkeit, Individualisierbarkeit, Steuerbarkeit, Lernförderlichkeit und Fehlertoleranz sind wichtige Prinzipien. Nach diesen Prinzipien bereiten De-

2 Michel, Antje, 2016. Informationsdidaktik - Skizze eines neuen informationsdidaktischen Forschungsfelds. In: Information - Wissenschaft \& Praxis, 67(5-6), Seiten 325-330. https://opus4.kobv.de/opu s4-fhpotsdam/frontdoor/index/index/docId/1812 [20.1.2020].

3 Tomitsch, Martin; Wrigley, Clara, 2018. Design. Think. Make. Break. Repeat. A Handbook of Methods. Amsterdam: BIS Publishers. signerinnen und Designer Informationen auf und stellen sie als App, Website, Social Media Kanal, Infografik, Lehrbuch, Exponat (z. B. in Museen) der Gesellschaft zur Verfügung. ${ }^{4}$ Was die nutzerzentrierte (Usability) und motivierende, attraktive (User Experience) Aufbereitung und Verbreitung von Informationen betrifft, analog und vor allem digital, könnte die Informationswissenschaft meines Erachtens viel enger mit den Design-Disziplinen kooperieren.

\section{Spannungsfeld „aktuelle Nutzung“ und „kulturelle Überlieferung“}

Antje Michel: Was Du beschreibst, ist eine Aufbereitung konkreter Informationen für die aktuelle Nutzung. Hier unterscheiden sich die Perspektiven des Designs - nicht absolut aber idealtypisch - und der Informationspraxis. Zum einen liegt die Perspektive der Informationspraxis, wie sie in Archiven, Bibliotheken, Dokumentations- oder Wissensmanagementeinrichtungen praktiziert wird, eher auf der Aufbereitung von Informationsbeständen, der Bildung, Kuratierung und Erhaltung von Sammlungen und der Erschließung und Nutzbarmachung eben dieser Sammlungen. Zum anderen bereiten Informationseinrichtungen zwar Ihre Sammlungen für die aktuelle Nutzung durch definierte Gruppen auf (da komme ich gleich nochmal drauf zurück). Aber von ihrer disziplinären Genese bilden sie Sammlungen für die Überlieferung unseres kulturellen Erbes. Sinnvolle Nutzung aus dieser Perspektive ist nicht an einer konkret und aktuell zu beschreibenden Zielgruppe zu definieren, sondern ist eine antizipierte Nutzung in einer aus Design-Perspektive sehr weit entfernten Zukunft von Jahrzehnten oder Jahrhunderten. Und wie entscheiden wir in der Gegenwart über die Relevanz bestimmter Informationen bzw. die „richtige“ Zusammensetzung definierter Sammlungen für eine zukünftige Nutzung unbekannter Nutzenden mit unbekannten Nutzungsanliegen? Archive, Bibliotheken und Dokumentationseinrichtungen legen hierfür vorrangig formale Kriterien zugrunde, wie z. B. den gesetzlichen Sammlungsauftrag, die Singularität der Information oder den Erhaltungszustand. Aber es gibt auch „weiche“ Faktoren, wie den „Informationswert“, der natürlich immer sehr subjektiv ist. Den Fokus auf die Formalkriterien halte ich für die langfristige Sammlung für absolut sinnvoll, denn er ist vermutlich am

4 Stickdorn, Marc; Schneider, Jacob, 2011. This is Service Design Thinking: Basics - Tools - Cases. Amsterdam: Bis Publishers. 


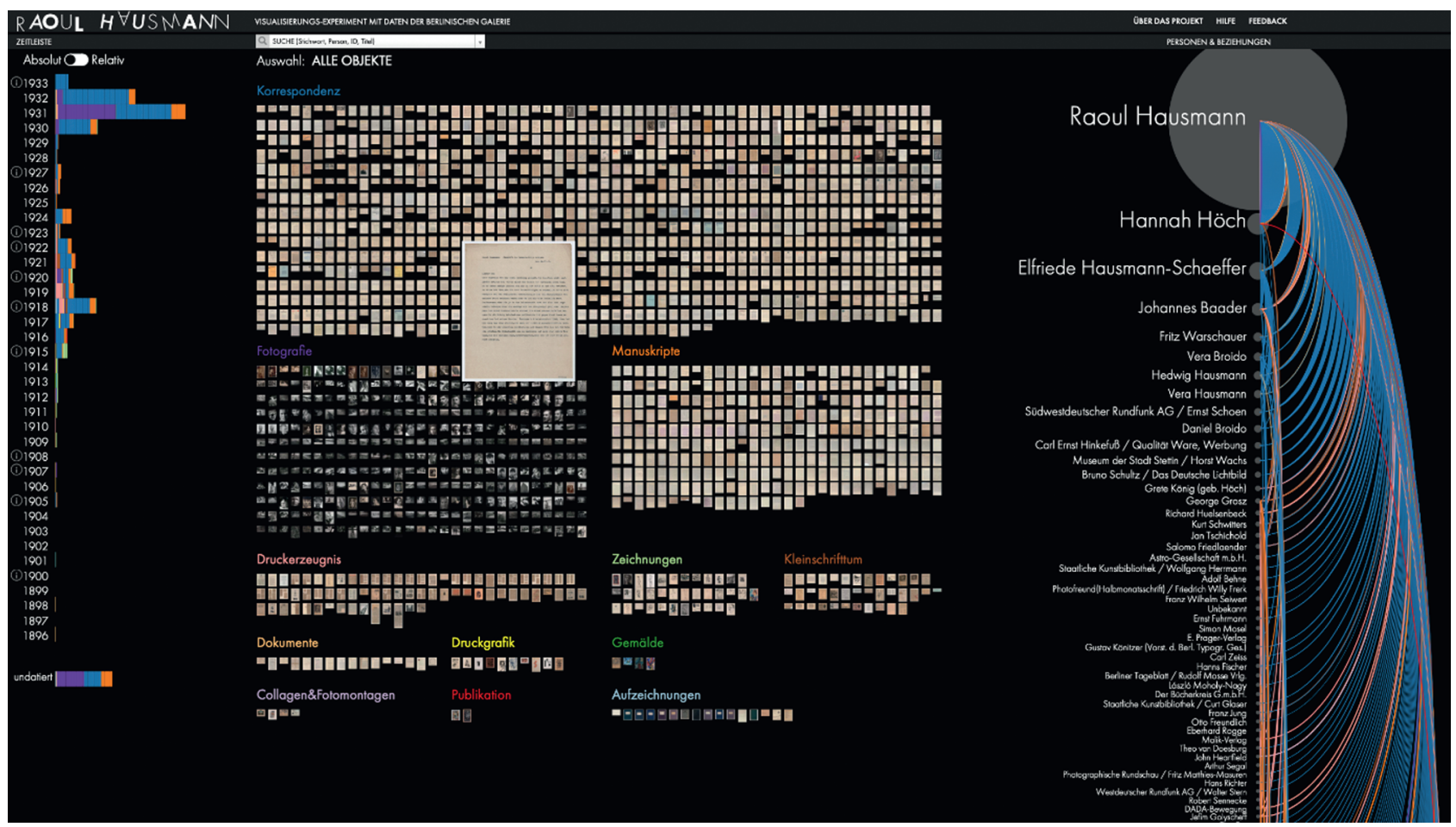

Abbildung 2: Screenshot User Interface Sammlung Raoul Hausmann (Berlinerische Galerie); Masterarbeit Mark-Jan Bludau, Fachbereich Design, FH Potsdam. Prototyp verfügbar unter: https://uclab.fh-potsdam.de/hausmann/. Letzter Abruf am 4.2.2020.

diskriminierungsneutralsten. Aber als Richtschnur für die Beurteilung des Informationswerts könnte ich mir durchaus vorstellen - und übrigens auch in allen anderen Feldern, in denen Nutzerinnen und Nutzer orientiert Services entwickelt werden -, dass Methoden aus den Design-Disziplinen, wie z.B. die Szenario-Technik zur Entwicklung alternativer Zukunftsvorstellungen oder die Persona-Methode zur Entwicklung möglichst unterschiedlicher Typen von Nutzerinnen und Nutzern eine sehr bereichernde Erweiterung des Methodenrepertoirs der Informationsberufe sein könnten.

So. Und jetzt springen mir vermutlich schon bereits 80 Prozent der Lesenden aus der Informationspraxis an den Hals, denn natürlich ist der aktuelle Bedarf auch eine sehr wesentliche Richtschnur für die informationspraktische Arbeit. In Bibliotheken, aus deren Perspektive ich als langjährige wissenschaftliche Bibliothekarin die Informationswissenschaft und ihre berufliche Praxis natürlich ganz stark sehe, gibt es ja z.B. die Unterscheidung zwischen Archivbibliothek und Gebrauchsbibliothek. Öffentliche Stadtbibliotheken oder Hochschulbibliotheken definieren sich vorrangig als Gebrauchsbibliotheken. Hier ist natürlich die Orientierung an der aktuellen Nutzung und an spezifischen Nutzendengruppen höchst relevant. Aus meiner Sicht, und das Thema bringen meine Kolleginnen der Fachgruppe Informationskompetenz der KIBA und ich seit einigen Jahren in den Fachdiskurs ein, wird bei der
Gestaltung von Bibliotheksservices viel zu stark von verallgemeinerbaren Standards ausgegangen und es werden zu wenig die sehr unterschiedlichen Praktiken des Umgangs mit Information und Wissen unterschiedlicher Wissenskulturen berücksichtigt. ${ }^{5}$ Und, ich stimme Dir voll zu, bei der User Experience können wir wirklich von den Design-Disziplinen lernen. Das zeigen ja auch Eure Projekte am Fachbereich Design, die Ihr zur Visualisierung kultureller Sammlungen durchgeführt habt. Das sind ganz wunderbare Beispiele für das Potenzial einer Zusammenarbeit der kuratierenden Informationspraxis, der DesignDisziplinen und der Informationswissenschaft.

\section{Interfacedesign als Instrument für informierte Datennutzung}

Frank Heidmann: Ja, der Screenshot zeigt eine prototypische Visualisierung, erstellt im Rahmen der Masterarbeit

5 Michel, Antje, Tappenbeck, Inka, 2019. Information Literacy, Epistemic Cultures and the Question "Who Needs What?" [Online]. In: M. Richter, Hg. LILG-2019. Conference Proceedings of the LILG 2019 Conference (2019). Frankfurt am Main, S. 1-10, https://informationli teracy.eu/conference/assets/papers/LILG-2019_Michel-Tappenbeck_ IL-epistemic-cultures.pdf [17.11. 2019]. 
„Perspektivbedingte Datenvisualisierungen“ in Zusammenarbeit mit der Berlinischen Galerie. Der Prototyp ist eine explorative Visualisierung der Daten und Bilder des Raoul Hausmann Nachlasses der Berlinischen Galerie und soll einen Einblick in die Vielfalt der Sammlung ermöglichen. In diesem Feld der innovativen Visualisierung kultureller Sammlungen haben wir tatsächlich schon viele spannende Projekte mit Informationseinrichtungen gemacht. Und da sind wir schon ganz nah an einer anderen Herausforderung, die unsere beiden Disziplinen trifft: Die Informationswissenschaft - meine Hoffnung und auch häufig so beschrieben ${ }^{6}$ - kann die Design-Disziplinen für einen noch reflektierteren Umgang mit den Themen Privatsphäre, Datensicherheit, Open Data etc. sensibilisieren; z.B. hinsichtlich der Notwendigkeit überhaupt (so viele) Daten zu sammeln, sie angemessen zu schützen, zu archivieren, zu löschen bzw. verantwortungsvoll zu teilen, d.h. der Öffentlichkeit - also der Wissenschaft, Wirtschaft oder Zivilgesellschaft - zur Verfügung zu stellen. Dass es dazu bestimmter Kompetenzen bedarf - Digital Literacy ist dann wieder ein zentrales Forschungs- und Praxisfeld beider Disziplinen: Um digitale Teilhabe, digitales Lernen, Datensouveränität etc. didaktisch sinnvoll zu vermitteln bzw. zu ermöglichen, sind neben methodischen Konzepten auch und vor allem innovative User Interfaces und Visualisierungen zur Förderung der immer wieder geforderten digitalen Souveränität notwendig. Darum geht es auch in Constanzes neuem Projekt, in dem Konzepte für „DataDashboards“, also Oberflächen zum Monitoring des eigenen Datenfußabdrucks entwickelt werden sollen, um Individuen bei Entscheidungen im Umgang mit ihren Daten zu unterstützen. Das gleich gilt für die erfolgreiche Umsetzung von Open Data, Open Science, Citizen Science, Scholarly discovery, Open scholarship, die ja auch wichtige Anliegen der Informationswissenschaft sind. Diese Paradigmen und Konzepte werden nur dann „die Gesellschaft“ erreichen, wenn die Interfaces, ob nun digital, analog, haptisch oder akustisch, über eine hohe Usability, Accessibility und User Experience verfügen. Und das wird nur zusammengehen: Design, Computer Science, Informationswissenschaft. $^{7}$

6 Leetaru, Kalev,2019. Computer Science Could Learn A Lot From Library And Information Science. Forbes. 05.08.2019, www.forbes.co $\mathrm{m} /$ sites/kalevleetaru/2019/08/05/computer-science-could-learn-alot-from-library-and-information-science/\#2617179e587d [20.1.2020]. 7 Simsarian, Kristian, 2019. Design Education Can Change the World. In: Interactions. 26 (2), Seiten. 36-43. DOI: 10.1145/3305362 [20.1. 2020].

\section{Intuitive Frontends benötigen gute Datenstrukturierung}

Antje Michel: Lustig, dass Du gerade Kalev Leetaru zitierst, denn er ist einer der wenigen Informationswissenschaftler, der von seinem sehr selbstbewussten und offensiven Habitus seiner Wissenschaftskommunikation auch ein Designer sein könnte. Und vielleicht ist das gerade ein guter Beleg für Deine implizit geäußerte These, die Informationswissenschaft schafft es nicht, ihre genuinen Themen, wie z. B. Daten- und Informationssicherheit, Datenund Informationsgeschäftsmodelle, Datenschutz und Informationsfreiheit angemessen für die unterschiedlichen Öffentlichkeiten aufzubereiten. Ja, ich merke natürlich schon bei uns in der Lehre, dass diese Themen als ausgesprochen trocken wahrgenommen werden. Nicht nur in unserem gemeinsamen Seminar im Design oder bei den Abschlussarbeiten, die ich mitbetreut habe, werden ziemlich unbekümmert Informationen in kostenfreien kommerziellen Systemen kollaborativ erarbeitet und Persönlichkeits- und Nutzungsrechte missachtet. Vielmehr ist das ein allgemeines Phänomen. Das ist übrigens ein Forschungsthema, das aus meiner Sicht sehr spannend an der Schnittstelle zwischen Designwissenschaft und Informationswissenschaft angesiedelt wäre: das Prinzip Autorinnen und Autorenschaft sowie seine Relevanz in der Remix-Culture. Und noch einmal zurück zu Deiner These, diese Themen könnten nur dann eine größere Öffentlichkeit erreichen, wenn die Interfaces entsprechend gestaltet sind - ja, auch da stimme ich Dir zu und hier sehe ich auch eine produktive Möglichkeit der Zusammenarbeit zwischen Design und Informationswissenschaft: denn Euer Fokus auf Usability, Accessibility und User Experience gestaltet das Front End der Interfaces. Dahinter liegt aber häufig auch das Back End einer riesigen Menge von Informationen. Ein gutes Beispiel sind die Discovery Services (für die von dir angesprochene „Scholarly discovery“, also die wissenschaftliche Recherche). In denen müssen Publikationen aus extrem vielen Quellen zusammengeführt werden, die hinsichtlich ihrer Metadaten wie auch ihrer Nutzungsmodelle sehr heterogen sind und aus deren Datenbasis bei einer Suchanfrage dann sinnvoll ausgewählt werden muss. Das funktioniert nur so gut, wie die dahinterliegenden Metadatenschemata es sind. Die Strukturierung von Information, die Systematik der Informationsanordnung werden häufig als Gegensatz zu einer eher als intuitiv empfundenen Usability dargestellt. Ich denke, das ist nicht richtig. Gute Retrivalsysteme benötigen beispielsweise immer beide Dimensionen. Ich könnte mir das tatsächlich als sehr interessante methodische oder eher epis- 
temologische Erweiterung der Design-Theorien vorstellen, zu versuchen, die Perspektive der Nutzendenzentrierung mit dem Prinzip der Klassifikation (sei es als hierarische Taxonomie oder als netzartige Ontologie) zu integrieren.

\section{Balance von Bequemlichkeit und Kompetenzverlust in der Digitalisierung}

Constanze Langer: Damit zusammen hängt ein weiteres Metathema, das wir zum Beispiel im Fachbereich Design in diversen Lehrveranstaltungen aufgreifen und das in der Scientific Community, vor allem im Bereich Human Computer Interaction, kontrovers diskutiert wird. Es berührt beide Disziplinen, Design und Informationswissenschaft im Kern: Nur weil Maschinen immer intelligenter werden, dürfen wir die komplizierten Dinge nicht ihnen überlassen. ${ }^{8}$ Wie gehen wir damit um, dass immer mehr Werkzeuge, wie intelligent auch immer, uns immer mehr Arbeit abnehmen und sich gleichzeitig unsere Kompetenzen beim Lesen / Textverständnis, Schreiben, Rechnen, Zeichnen, Weltwissen insgesamt, Raumverständnis (mental maps) etc. verschlechtern? Wieviel „Convenience“ zum Beispiel beim Informationserwerb durch Apps wie get abstract oder Blinkist oder bei der Kommunikation durch Tweets und Instagram-Stories oder im Kontext der digitalen Lehre oder von algorithmischer Gestaltung ist verträglich für die Gesellschaft und vor allem für ihre Resilienz gegenüber den negativen Folgen der Digitalisierung und der Entstehung von immer mehr „Dumb-Smart Technologies". 9

Antje Michel: Die Frage, inwieweit die Bequemlichkeit durch immer hilfreichere (digitale) Werkzeuge unser Knowing-how und unsere Kulturpraktiken verkümmern lässt, ist wirklich wichtige Frage, finde ich. Ich muss ganz ehrlich sagen, meine Sichtweise darauf ändert sich momentan andauernd. Wir haben ja in der gesamten Kulturgeschichte immer schon Fähigkeiten und Fertigkeiten verloren, wenn eine spezifische Innovation diese überflüssig hat werden lassen und zunehmend übernehmen tech-

$8 \mathrm{Wu}$, Tim, 2018. The Tyranny of Convenience. The New York Times. 16.02.2018. https://www.nytimes.com/2018/02/16/opinion/sunday/ tyranny-convenience.html [18.1.2020].

9 Schöning, Johannes, 2019. Finsterworlds: Bringing Light into Technological Forests Through User Empowerment. In: Interactions 26 (6), Seiten 6-7. DOI: 10.1145/3365215. [20.1.2020]. nische Artefakte die Handlung, mit der wir unser Handlungsziel erreichen. Das fängt mit so einfachen Dingen an, wie dem Feuerzeug und dem Resultat, dass nur noch Menschen mit Pfadfinderinnen und Pfadfinder-Vergangenheit ohne Feuerzeug Flammen entfachen könnten. Und natürlich haben auch die von dir genannten Kulturtechniken, wie z.B. die Schrift schon eine Degeneration hinter sich, z.B. durch die Erfindung des Buchdrucks und die damit verbundene Reduktion der Handschrift um einen großen Teil ihrer künstlerischen Ausdrucksform. Man könnte jetzt also sagen, das ist normal. Außerdem gibt es bisher tatsächlich hinsichtlich der Fähigkeit des Schreibens keine Forschungsergebnisse, die eindeutig belegen dass der Umgang mit digitaler Technik die Fähigkeit, zu schreiben verschlechtert. Das liegt natürlich daran, dass Schreiben eine Fähigkeit ist, die sich aus ganz vielen unterschiedlichen Komponenten zusammensetzt und - gemäß dem heutigen Forschungsstand - werden einige eher gefördert durch die Rezeption und Produktion digitaler Texte und andere möglicher Weise nicht. ${ }^{10}$ Ist es also problematisch, wenn die Kulturtechnik des Schreibens sich noch weiter transformiert? Von der kunstvollen Handschrift (die übrigens nur vergleichsweise wenige Menschen beherrschten), über die Schreibschrift (deren weite gesellschaftliche Verbreitung ein Effekt und ein Instrument der Demokratisierung des Wissenszugangs ist) zum Schreiben mit der Tastatur oder gar durch Sprachsteuerung? Auf der anderen Seite ist natürlich die Frage, ob es eine Art „Kipppunkt” gibt. Wann wir also nicht mehr fähig wären, unsere kulturelle Identität als Mensch zu leben, durch ein Ereignis, das uns die nötigen Werkzeuge raubt und wir auf eine analoge Lebenspraxis zurückgeworfen, gesellschaftlich nicht mehr in der Lage wären, diese auszuüben. Nicht mehr ohne digitale Assistenz schreiben, zeichnen, uns orientieren oder auch kreativ sein könnten.

\section{Artificial Intelligence und die Zukunft des Designs}

Frank Heidmann: An dieser Frage hängt natürlich das ganze AI-Thema, das für beide Disziplinen weitreichende Folgen haben wird - oder auch nicht, oder ganz anders, als jetzt in den vielen Studien prognostiziert wird. AI (Artificial Intelligence) und die verwandten, aber nicht iden-

10 Bulut, Necle, 2019. Faktencheck. Handschrift in der digitalisierten Welt. Köln. S. 4-5, https://www.mercator-institut-sprachfoerderung. de/de/publikationen/faktenchecks/ [18.1.2020]. 
tischen Themen Machine Learning und Deep Learning werden sowohl zukünftige Gestaltungsprozesse, als auch Nutzungserlebnisse radikal verändern. Uns Designerinnen und Designer betrifft diese Entwicklung von beiden Seiten: „Designing with AI“ wird den kreativen Arbeitsprozess massiv verändern und auch die Nutzung unserer Services und Produkte wird sich durch AI-Interaktionen wandeln. Das ist zumindest die einhellige Prognose der großen Technologiefirmen, Tech-Online-Nachrichtenportale und immer häufiger auch Mainstream-Medien mit mehr oder weniger differenzierter Reflexion der gesellschaftlichen Folgen dieser Entwicklung.

Unabhängig von den großen Diskurslinien im AI-Kontext, wie dem drohenden massiven Arbeitsplatzverlust oder Ängsten, Maschinen könnten eines Tages Selbstbewusstsein erlangen und den Menschen verdrängen, existieren bereits eine ganze Reihe technologischer Entwicklungen, die Erlebnisse und Erfahrungen grundlegend verändern können. Aus der Designperspektive werden derzeit vor allem folgende Fragestellungen im Spannungsfeld AI-Design thematisiert:

1. das „Aufbrechen der Black Box AI“, d.h. die angemessene Erfahrbarmachung, z.B. Visualisierung, von algorithmischen Prozessen für Nicht-Experten mit dem Ziel Vertrauen zu schaffen und / oder ein erfolgreiches Eingreifen des Menschen bei einem wie auch immer gearteten Versagen von AI sicher zu stellen. Die Anwendungsszenarien umfassen dabei viel mehr als das in diesem Zusammenhang immer wieder zitierte autonome Fahren. Sie erstrecken sich auf alle möglichen Kooperationsformen mit lernenden Systemen in nahezu allen Lebensbereichen.

2. Die „radikal personalisierte User Experience“ oder Services, die über die Auswertung von individuellen Sensordaten durch neuronale Netzwerke etc. Vorhersagen über das Verhalten der Nutzerinnen und Nutzer ermöglichen, und für die angemessenen Formen der Darstellung, Korrektur und der Akzeptanz gestaltet werden müssen. Dies ist nicht zuletzt auch eine Informationsethische Fragestellung.

3. Die Entwicklung von kreativen Zukunftsvisionen mit „Critical Design Ansätzen für AI-Systeme“, die über aktuell akzeptierte und für die nähere Zukunft realisierbare Produkte hinausgehen und alternative smarte Zukünfte jenseits heutiger Visualisierungen entwickeln und zur Diskussion stellen.

4. Der Einsatz von AI in „Virtual Reality- \& Augumented Reality-Systemen“, die natürliche künstliche Welten erschaffen und sukzessive lernen, dem Nutzer oder der Nutzerin kontextsensitiv immer passgenauer Informationen handlungsleitend zur Verfügung zu stellen.
5. Das alles führt natürlich zu der Frage nach den zukünftigen Kompetenzprofilen für Designerinnen und Designer: Hier können nur mit Methoden der $\mathrm{Zu}$ kunftsforschung Visionen zur Frage entwickelt werden, wie AI Gestaltungsprozesse und damit die Ausbildung und das Skill-Set von Gestalterinnen und Gestaltern verändern wird. Eine ganz wesentliche Frage, mit der sich auch unsere Studierenden auseinandersetzen, ist: an welchen Stellen ersetzt zukünftig algorithmische Gestaltung den menschlichen Gestalter bzw. die Gestalterin?

\section{Al als Herausforderung und Chance für Informationswissenschaft und -praxis}

Antje Michel: Ja. Der ubiquitäre Zugang zu Information mithilfe des Internets hat auch zu einer existenziellen Krise von Informationseinrichtungen, v. a. Bibliotheken und Dokumentationseinrichtungen, in weniger hohem Maße von Archiven geführt (und diesen Institutionen gleichzeitig unglaublich viele neue Möglichkeiten beschert). Bei den Bibliotheken zum Beispiel, führte die Konkurrenz im Kerngeschäft „Zugang zu Information“ durch immer besser werdende Suchmaschinen $\mathrm{zu}$ manchmal regelrecht verzweifelt wirkenden Neuerfindungen. Die Bibliothek ist „dritter Ort“, sie ist „Community Managerin“, und vor allem natürlich „Lotsin durch die Informationsflut”. Und jetzt AI: Google hat 2015 seinen auf AI basierenden Algorithmus Rank Brain eingeführt. Es ist nur einer unter vielen, der die Gestaltung einer Trefferliste letztlich bestimmt. Aber er hat dazu geführt, dass plötzlich auch qualifizierte Treffer auf vorher unbekannte Anfragen angeboten werden konnten. ${ }^{11}$ AI nimmt in der Suchmaschinentechnologie also inzwischen schon recht erfolgreich eine Lotsinnenfunktion durch die Informationsflut ein. Je mehr Rank Brain dazu lernt, desto weniger relevant wird möglicherweise noch die bibliothekarische Beratung sein - auch in hochspezialisierten Wissensbereichen, in denen ein Großteil der Informationsanfragen einzigartig sind. Die Nutzenden empfinden bei der Informationssuche im Internet eine Ambivalenz: einerseits ist die Performance einer Internetrecherche in der Regel hoch, andererseits nehmen sie „Kollateralschäden“ des vernetzten Umgangs

11 Wells, Tom, 2015. RankBrain, künstliche Intelligenz und die Entwicklung der Google-Architektur [Blog-Beitrag]. https://blog.searchme trics.com/de/rankbrain/?_ga=2.222899231.520205034.1579369565-264 583562.1579369565. [18.1.2020]. 
mit Ihren Daten wahr, deren Logik sie nicht verstehen und die sie verunsichert. ${ }^{12}$ Hier sehe ich eine ganz wichtige Aufgabe der Informationswissenschaft, Konzepte zur Beurteilung von Information im Zeitalter von vernetzten und maschinell ausgewerteten Daten zu entwickeln. Und die Einrichtungen der Informationspraxis müssen sich intensiv darauf einstellen, derartige Konzepte an ihre Nutzerinnen und Nutzer zu vermitteln.

Informationswissenschaftlich und auch informationspraktisch ist AI natürlich super spannend. Die Deutsche Gesellschaft für Information hat sich z. B. letzten Herbst auf einer Tagung mit dem Thema Digitalisierung und Lernen auseinander gesetzt. ${ }^{13}$ AI verfeinert jetzt schon die Möglichkeiten der OCR-Texterkennung und der Strukturieren bei Digitalisierungsprojekten. Die Staatsbibliothek Berlin nutzt AI darüber hinaus z.B. zur Verbesserung der Eigennamenerkennung (Named-entity recognition) in historischen Digitalisaten. ${ }^{14}$ Der Einsatz von AI in derartigen Verfahren bietet großartige Möglichkeiten im Bereich der digitalen Geisteswissenschaften und verändert die Services aber auch die Rolle von spezialisierten Bibliotheken in diesem Feld. Bibliotheken werden zu aktiven Partnerinnen in der Forschung. Möglicher Weise finden Bibliothekspraxis und Informationswissenschaft in diesen forschenden Praxisfeldern wieder näher zueinander. Auf einer Meta-Perspektive ganz augenfällig ist natürlich der informationswissenschaftliche Bezug auch bei Fragen der Informationsethik, womit sich z.B. der britische Informationsphilosoph Luciano Floridi auseinander setzt. Aber auch in meinem Forschungsfeld, der Informationsdidaktik des Wissenstransfers wird es spannende neue Fragestellungen geben, z.B. wie Wissenstransfer didaktisch geplant werden kann, wenn Entitäten in der Kommunikationskette lernende Maschinen sind, deren Informationsverhalten wir nicht mehr verstehen.

12 Head, Alison J., Fister, Barbara, MacMillan, Margy, 2020. Across the great divide: How today's college students engage with news (No. 8). Cambridge, Massachusetts: Harvard University. 10.5210/fm. v24i8.10166. [20.1.2020].

13 Deutsche Gesellschaft für Information und Wissen e.V., 2020, DGI-Forum Wittenberg 2019 - Programm. Frankfurt am Main: DGI, 20.01.2020, https://dgi-info.de/dgi-forum-wittenberg-2019-program $\mathrm{m} /$ [20.1.2020].

14 Staatsbibliothek zu Berlin - Preußischer Kulturbesitz, Clemens Neudecker, 2019. Kuratieren mit KI: Erste Ergebnisse aus dem QURATOR-Projekt - SBB aktuell [Blog-Beitrag vom 4.6.2019], https://blog.s bb.berlin/zwischenergebnisse-qurator-jahr-1/ [20.1.2020].

\section{Neue Methoden für die Informationswissenschaft?}

Frank Heidmann: Vielleicht wäre das mal eine reizvolle Fragestellung für den Einsatz der Methode „Design Fiction“ bzw. „Speculative Design“. ${ }^{15}$ Das ist eine Methode, die ich für beide Disziplinen als Erweiterung des Berufsfeldes interessant finde: Zahlreiche wissenschaftliche Disziplinen beschäftigen sich mit der Vorausschau auf zukünftige gesellschaftliche Entwicklungen. Forschungsrichtungen wie die Risikoforschung, die Technologiefolgenabschätzung oder wissenschaftliche Zukunftsforschung widmen sich ausschließlich bzw. überwiegend der wissenschaftlichen Auseinandersetzung mit Zukunftsfragen. Wie Popp in einer aktuellen Sammlung von Science Fiction-Erzählungen konstatiert, gibt es in der Logik des Wissenschaftsverständnisses dieser Disziplinen eine nicht überschreitbare Demarkationslinie zwischen Science und Science Fiction. ${ }^{16}$ In den künstlerischen und gestalterischen Disziplinen dagegen, werden fiktionale Formate häufig zur Zuspitzung und zum „Re-Framing“ von Problemstellungen, also zum Perspektivwechsel, eingesetzt. Design wird als ein Mittel des hypothetischen und spekulativen Fragens benutzt, um auf diese Weise herauszufinden, wie technologische und gesellschaftliche Entwicklungen (z. B. Mobilität- und Konsumkulturen) auf andere Weise existieren und funktionieren könnten. Das Spektrum beginnt bei inzwischen schon fast überall eingeführten Formaten wie dem „Design Thinking“ und endet bei „Design Fiction“, „Speculative \& Critical Design“ und ähnlichen Formaten, die immer stärker auch Einzug in andere Disziplinen und die Wirtschaft finden. Was meinst Du, Antje, wie könnte die Informationswissenschaft und ihre Curricula sich stärker auch an diesen Methoden alternativer Wissenssysteme bedienen, um ihren originären Methodenkanon zu erweitern, bzw. an die Komplexität und Beschleunigung der diversen Entwicklungen in der Gesellschaft anzupassen?

Antje Michel: Ich denke, die Methoden der Design-Disziplinen bieten ein großes Potenzial für die Informationswissenschaft und wir haben ja auch bereits einige Anwendungsmöglichkeiten besprochen. Es sind aber nicht nur

15 Dunne, A., Raby, F. (2013): Speculative Everything. Design, Fiction, and Social dreaming. Cambridge, MA: The MIT Press.

16 vgl.: Popp, Reinhold, 2019. Zukunftsdenken in Literatur und Wissenschaft. In: S. Brandt, C. Granderrath, M. Hattendorf (Hrsg.) 2029. Geschichten von morgen. Berlin: Suhrkamp. Seiten 521-536. 
1.
Plan the human-centred design process.

\section{Human-Centred Design Process}

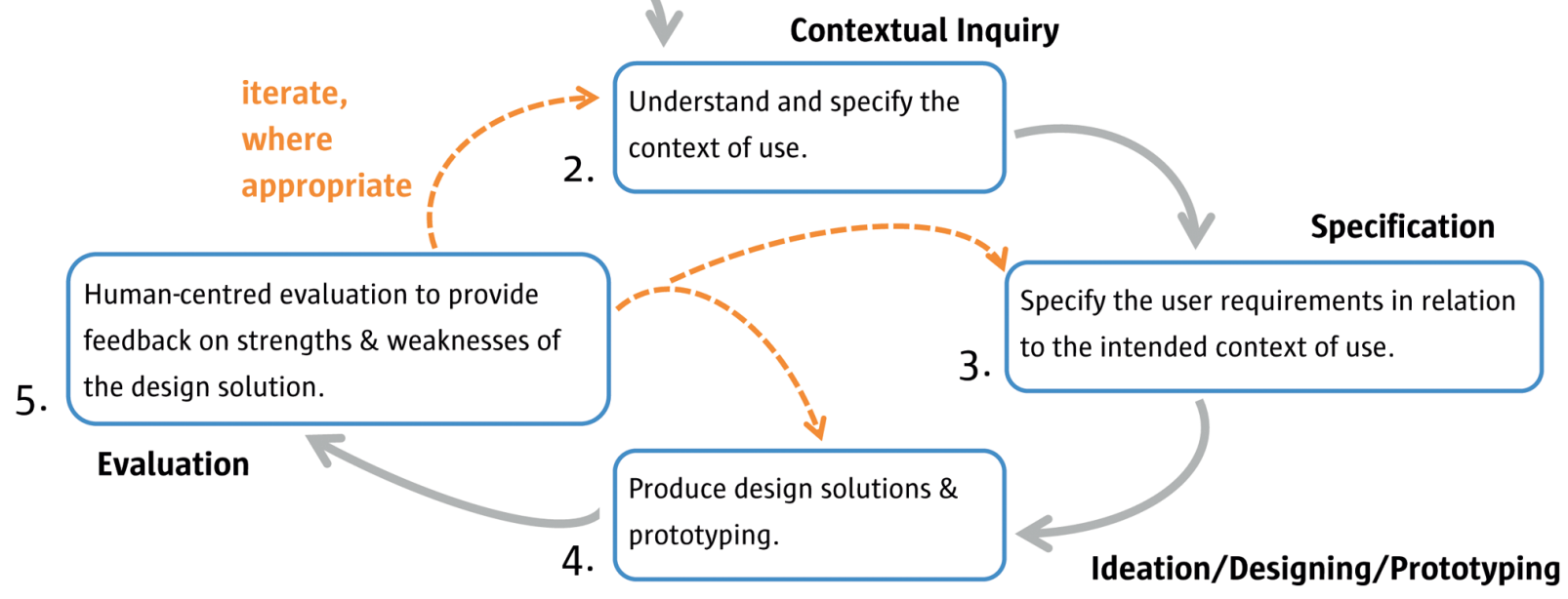

Abbildung 3: Idealtypischer nutzerinnen- und nutzerzentrierter Designprozess auf Grundlage der ISO-Norm 9241-210:2010 „Ergonomics of human-system interaction - Part 210: Human-centred design for interactive systems“. (Quelle: FH Potsdam)

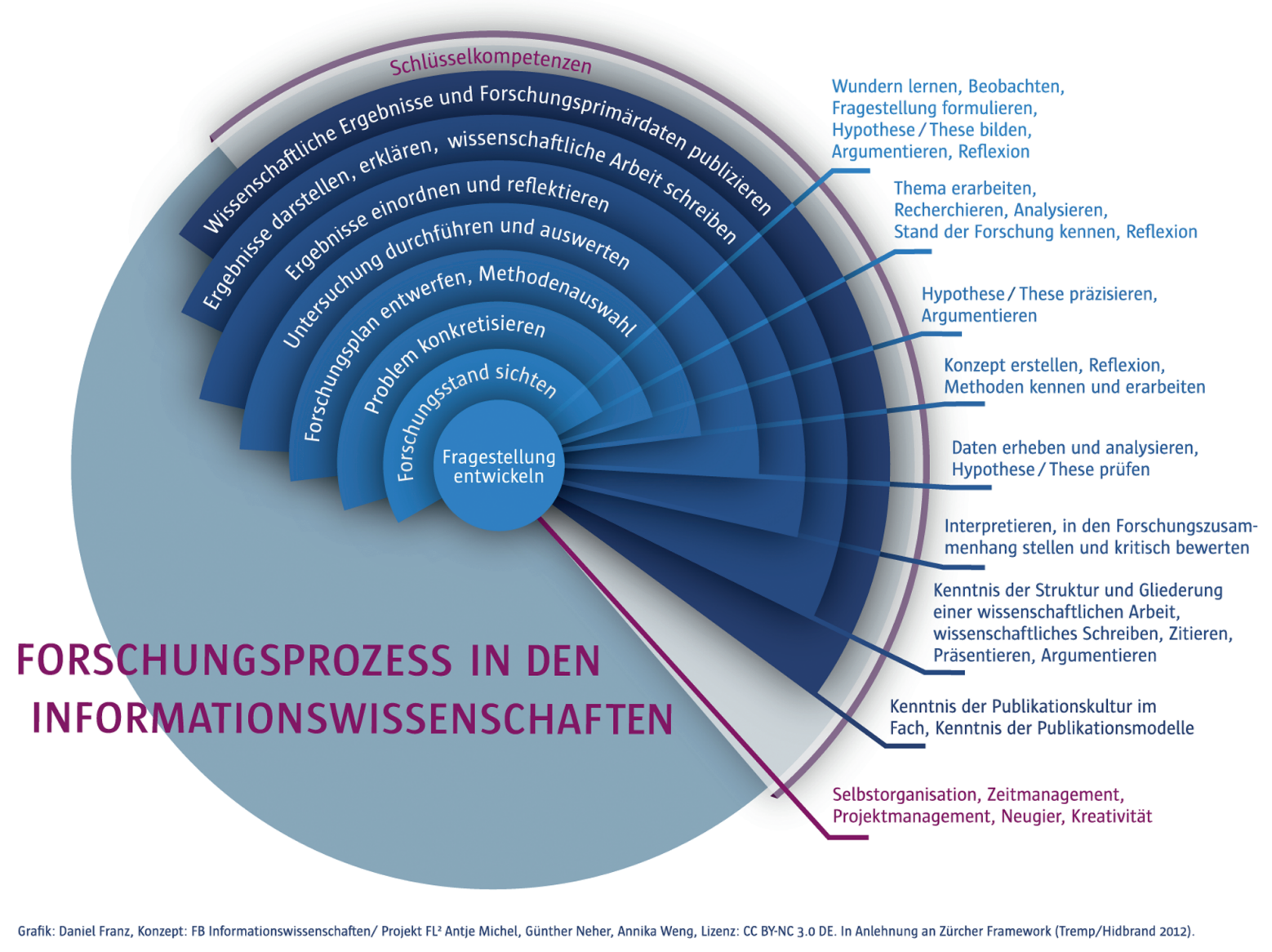

Abbildung 4: Idealtypischer informationswissenschaftlicher Forschungsprozess. (Quelle: FH Potsdam) 
die Methoden, sondern eigentlich mehr die gesamte Problemlösungsstrategie in den Design-Disziplinen. Wir haben ja in unseren Diskussionen häufig einander ,aufskizziert“, wie ein idealtypischer Erkenntnisprozess in der Informationswissenschaft und wie ein ebenso idealtypischer Entwurfsprozess in den Design-Disziplinen abläuft. Ich finde Eure stärker iterative, fehlertolerante und Nutzende orientierte Wissensgenerierungspraktik für die vielfach ja total praktischen Probleme, die in den Informationsberufen zu lösen sind, häufig viel angemessener, als das stark am empirischen Forschungsprozess orientierte Vorgehen, das unseren Problemlösungsstrategien zugrunde liegt.

Aber das müssten wir dann wirklich in die Curricula unserer Studiengänge integrieren - und das ist ja teilweise auch schon der Fall, wenn die Lehrenden sich methodisch an den Entwicklungsprozessen der Informatik orientieren. Was ich dagegen eher kritisch sehe, ist die in den Informationsberufen verbreitete Auffassung, man könne ein Handbuch lesen oder einige Workshops besuchen und dann das Mindset bzw. die Methodik einer anderen Disziplin einfach mal übernehmen. Da bleibt viel auf der Strecke. Das sehe ich sowohl bei der Adaptation der Mediendidaktik im bibliothekarischen Kontext als auch in Bezug auf die neuerdings stark im Trend liegenden Design Thinking Workshops. Im Zweifel bin ich dann eher dafür, ein bestimmtes Problem in interdisziplinärer Kooperation zu lösen als den Anspruch zu haben, die Expertise der anderen Disziplin voll selber abbilden zu können.

Deskriptoren: Methoden, Informationswissenschaft, Informationspraxis, Design, Digitalisierung

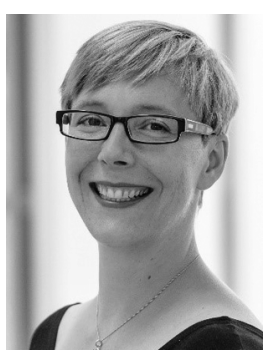

\section{Prof. Dr. Antje Michel}

Fachhochschule Potsdam

Fachbereich Informationswissenschaften

Kiepenheueralle 5

14469 Potsdam

michel@fh-potsdam.de
Prof. Dr. Antje Michel ist seit Januar 2016 Professorin für Informationsdidaktik und Wissenstransfer an der FH Potsdam. Sie studierte Soziologie in Göttingen und Berlin Soziologie und promovierte 2003 am Institut für Soziologie der Freien Universität Berlin. Im Anschluss an das Referendariat für den wissenschaftlichen Bibliotheksdienst arbeitete sie als wissenschaftliche Bibliothekarin an der Max Planck Digital Library und als Leiterin zweier großer Fachbibliotheken der Universitätsbibliothek der LMU München. Der zentrale Schwerpunkt ihrer Forschungs- und Lehrtätigkeit an der FH Potsdam liegt in der Entwicklung des international neuen Forschungsfelds der Informati- onsdidaktik sowie der interdisziplinären Integration informationsdidaktischer Konzepte und Forschungsergebnisse in die akademische Lehre und den Wissenstransfer.

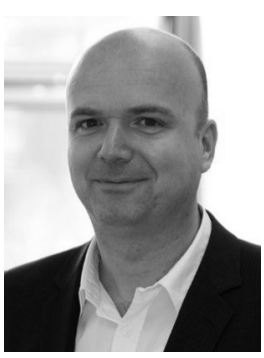

Prof. Dr. Frank Heidmann

Fachhochschule Potsdam

Fachbereich Design

Kiepenheueralle 5

14469 Potsdam

heidmann@fh-potsdam.de

Prof. Dr. Frank Heidmann arbeitete nach seinem Studium der Angewandten Physischen Geographie und Kartographie als Leiter des Forschungsbereichs »Human-Computer Interaction « am FraunhoferInstitut für Arbeitswirtschaft und Organisation (IAO). Seit 2005 ist er Professor für »Design of Software Interfaces « am Fachbereich Design der Fachhochschule Potsdam. Seine Arbeitsschwerpunkte liegen in den Bereichen Human-Centred Design, geographische Informationsvisualisierung sowie der Entwicklung nachhaltiger Smart City Services.

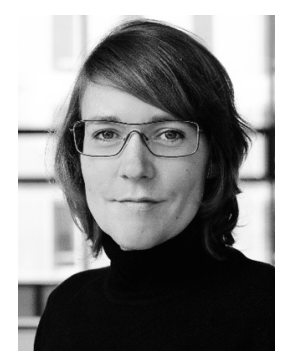

\section{Prof. Constanze Langer}

Fachhochschule Potsdam Fachbereich Design

Kiepenheueralle 5

14469 Potsdam

langer@fh-potsdam.de

Prof. Constanze Langer ist seit 2012 Professorin für Visual Interface Design und aktuell Dekanin des Fachbereich Design der Fachhochschule Potsdam. Sie studierte Industrial Design mit Schwerpunkt Interfacedesign sowie Interaction Design an der Hochschule Magdeburg-Stendal und der Hochschule Genk in Belgien. Als UI/UXDesignerin arbeitete sie für internationale Unternehmen in der Software- und Hardwareentwicklung sowie mit dem Schwerpunkt Infografik und Wissenschaftskommunikation für Forschungsinstitute wie die Max Planck Gesellschaft und diverse Fraunhofer Institute. Besonderes Interesse in der Forschungs- und Lehrtätigkeit liegt in der Analyse und der Visualisierung von Bedienprozessen, Informationsarchitekturen und Grafischen User Interfaces. 